\title{
Letters
}

Website: bmj.com

Email: letters@bmj.com

\section{Promoting patient safety in primary care}

\section{Honesty and openness may not be best policy}

EDITOR-We were both surprised by Wilson et al who, in their editorial, ask everybody to send in reports of their errors. ${ }^{1}$ Our concerns remain regardless of whether or not such accounts are published anonymously. After all, the police have used anonymous medical research data in their investigations. A conviction of culpable and reckless behaviour was secured against a man after he had sexual intercourse with his girlfriend without telling her he was infected with HIV. The scientific evidence that secured the conviction came from confidential research data that were obtained with a police warrant.

Furthermore, Wilson et all say that for doctors to report their errors, patient consent will be needed. This means that the patient will know that an account of his or her care is to be published and may read it. He or she may ask his lawyers to read it. The lawyers may then be able to substantiate this published account as a confession of guilt or negligence.

Wherever and whoever we are, we must be careful as doctors what we say. For example, although we understand that no charges

\section{Advice to authors}

We prefer to receive all responses electronically, sent directly to our website. Processing your letter will be delayed unless it arrives in an electronic form.

We are now posting all direct submissions to our website within 24 hours of receipt and our intention is to post all other electronic submissions there as well. All responses will be eligible for publication in the paper journal.

Responses should be under 400 words and relate to articles published in the preceding month. They should include $\leqslant 5$ references, in the Vancouver style, including one to the BMJ article to which they relate. We welcome illustrations.

Please supply each author's current appointment and full address, and a phone or fax number or email address for the corresponding author. We ask authors to declare any competing interest. Please send a stamped addressed envelope if you would like to know whether your letter has been accepted or rejected.

Letters will be edited and may be shortened.

bmj.com

letters@bmj.com were brought, an incident last year highlights this point. Police are to hold a murder inquiry into the case of Peter Brand, a Member of Parliament for the Liberal Democrat Party for the Isle of Wight and a general practitioner, who mentioned during a parliamentary debate that he had withdrawn treatment from a two year old boy with leukaemia at the parents' request, to save him any more distress. ${ }^{3}$ This case had occurred in 1973, when he was a house officer.

However regrettable, we do live in a culture of blame. To admit professional mistakes of a minor nature may be straightforwardly forgivable. The admission, however of a serious offence or negligence by doctors may result in litigation against them. Before discussing such matters in public, doctors should seriously consider whether honesty and openness are the best policy.

Graham Ness clinical lecturer in forensic psychiatry g.j.ness@sheffield.ac.uk

Christopher Cordess chair of academic section of forensic psychiatry

School of Health and Related Research, Sheffield University, Sheffield S1 4DA

1 Wilson T, Pringle M, Sheikh A. Promoting patient safety in primary care. BMJ 2001;323:582-3. (15 September.) 2 Dyer C. Use of confidential HIV data helps convict former prisoner. $B M J$ 2001:322:633.

3 prisoner. BMJ 2001;322:633. 2000;320:464.

\section{Practices should set up their own critical incident reporting}

EDITOR-The editorial by Wilson et al on promoting patient safety in primary care serves as a prompt to develop appropriately designed incident reporting systems. ${ }^{1}$ The publication of Organisation with a Memory and Doing Less Harm ${ }^{3}$ and the recent launch of the National Patient Safety Agency have firmly set the agenda for risk management in both secondary and primary care.

Learning to identify and manage our risks in primary care could be seen as an opportunity to enhance and focus continuing professional development, team working, and cultural change. Cultural change should be characterised, in part, by risk awareness, openness, and the opportunity for anonymous and blame free risk and incident reporting within our organisations, by any team member.

Sheikh and Hurwitz have highlighted the importance of developing primary medical error databases. ${ }^{4}$ This should come from within the profession if we are to incul- cate a sense of realism and ownership in practices in readiness for mandatory incident reporting.

In our practice colleagues and I have classified 25 types of adverse event. These include administrative and organisational, communication, and health and safety events and a range of clinical events (including diagnostic, therapeutic and prescribing, procedural, and case management "errors"). This is underpinned by a reporting system that takes account of the reporter's narrative and can be anonymous if required. Events are coded and stored on a secure computer, with the classified events recorded on dated spreadsheets with hyperlinks to the reporter's narrative, the analysis of root causes, and necessary actions as text files.

This will be enhanced by a more sophisticated database in due course, but this relatively simple approach has enabled us to get started by using our existing office software. This was relatively easy; the real work will lie in developing the culture and making the changes to reduce our risks and improve patient care. But if we can do it anyone can.

Steve Ruffles general practitioner

Portland Medical Practice, Aldridge, West Midlands WS9 8NS

RufflesS@gp.walsall-ha.wmids.nhs.uk

1 Wilson T, Pringle M, Sheikh A. Promoting patient safety in primary care. BMJ 2001;323:583-4. (15 September.)

2 Department of Health. Organisation with a memory. London: $\mathrm{DoH}, 2000$.

3 Department of Health, National Patient Safety Agency. Doing less harm. London: DoH, 2001

4 Sheikh A, Hurwitz B. Setting up a database of medical Sheikh A, Hurwitz B. Setting up a database of medical
error in general practice: conceptual and methodological considerations. BrJ Gen Pract 2001;51:57-60.

\section{Should NHS patients be allowed to pay extra for their care?}

\section{Patient payments bring new resources into system}

EDITOR-Richards et al in their article raise the issue of patients contributing to payments for cancer drugs in Britain's NHS. ${ }^{1}$ It is pleasing to see that times are changing for ethics committees even if the pervasive tone of self righteousness is a bit grating. If British politicians cannot manage to improve the NHS, why should a patient not pay for drug treatment perceived as beneficial? Would the group consider it unethical for a patient to pay for vitamin pills, special diets, alternative medicine, or a second opinion?

Over the next three years there is going to be a major shift in cancer care. New 
molecularly targeted medicines that block specific signalling pathways and angiogenesis will become available. These will take the form of simple tablets, to be taken once a day. They will prolong survival by a few months in many cancers, but as the predictive power of genomics and proteomics bites, cancer will become a chronic, controllable disease. The cost will exceed the budget of Britain's national cancer plan, which is just a catching up exercise on 30 years of underinvestment by successive governments. In a global society no group of armchair ethicists sitting in Nottingham can stop people ordering these new drugs through the internet and paying with their credit card. Debating the issue is futile.

What we can do is to ensure that the core cancer services offered to everyone by the NHS improve. The cancer plan provides an excellent structure but is underfunded. Creating the new bureaucracy has provided excellent spin for politicians who think they have "done cancer." The reality is grimageing radiotherapy machines, clear examples of continuing postcode prescribing, overworked staff, and lack of consultation time. Allowing patients to pay for treatments outside the core is one way of bringing additional resources into the system. Another is to privatise their delivery.

Karol Sikora visiting professor of cancer medicine Hammersmith Hospital, London W12 0HS karolsikora@hotmail.com

1 Richards C, Dingwall R, Watson A. Should NHS patients be allowed to contribute extra money to their care? $B M$ 2001;323:563-5. (9 September.)

\section{Debate is essential, not futile}

EDITOR-Richards et al raise many ethical and legal issues, discussing whether or not NHS patients should be allowed to contribute extra money to their care. ${ }^{1}$ In his rapid response and the letter above Sikora argues that debating the issue is futile. ${ }^{2}$ I believe that extensive debate by all stakeholders in the NHS is essential. This new class of "active" patient, wealthy enough to purchase expensive pharmacogenomic drugs such as Herceptin (trastuzumab), could add a new, unbalancing component to shared decision making, by rendering clinicians scarcely more than technicians. The effect on the research process would do a disservice to fellow patients with breast cancer and be disruptive to equitable and evidence based provision of health care in general in a health provision system that is based on egalitarian principles such as we have in the United Kingdom.

An editorial in the Lancet described the political activism of the national breast cancer coalition in the United States that, according to its president, brought Herceptin to women two years earlier than this would have happened without its advocacy. ${ }^{3}$ This attitude may bring benefits to its members but does not redress the balance of power in the research community. ${ }^{4}$ The conduct and quality of research globally would, however, be further diminished by ad hoc personal purchasing. Cultural differences and different systems of healthcare provision-for example between the United States and the United Kingdom-would probably result in even more divisive availability of treatments.

Furthermore, patients who have purchased such an expensive drug are not likely to agree to its administration only within the restraints of a trial protocol, where informed consent to test for eligibility and participation in the trial will be required. Trials are needed to establish the drug's efficacy in patients with early breast cancer. Will patients who purchase Herceptin also be allowed to sidestep testing for HER2 expression, which is necessary to determine eligibility for its use (where only $25-30 \%$ of primary breast cancers express the HER2 protein), thereby avoiding the discriminatory potential of the genetic test (which is only $80 \%$ accurate) for this type of breast cancer, for which the prognosis is worse? $?^{5} \mathrm{It}$ will make the treatment of breast cancer even more discriminatory if there is a further subdivision of patients who can either pay for it or pay for it and receive it outside of the context of a clinical trial, thus delaying production of data even longer. Problems of consent and information provision to participants of prospective trials will be particularly difficult with this new class of drugs, without the added problem of ad hoc purchasing.

Hazel Thornton independent advocate for quality in research and healthcare

Saionara, 31 Regent Street, Rowhedge, Colchester CO5 7EA

hazelcagct@aol.com

1 Richards C, Dingwall R, Watson A. Should NHS patients be allowed to contribute extra money to their care? $B M$ 2001;323:563-5. (9 September.)

2 Sikora K. Copayments for cancer drugs. Electronic response to Should NHS patients be allowed to contribute extra money to their care? bmj.com 2001;323 (bmj.com cgi/eletters/323/7312/563\#16524).

3 Anonymous. How consumers can and should improve clinical trials Lancet 2001:357:1721.

4. Thornton T Consumers and trials. Lancet 2001:358.763.

5 Peres J. Test problems flaw breast cancer care. Chicago Tribune 2001 Jul 2.

\section{Pragmatism should rule, not policy committees}

EDITOR-The approach at our cancer centre has been different to that reported in the paper by Richards et al. ${ }^{1}$ For non-funded cancer drugs, patients have been advised that they may continue under the care of their NHS consultant, who will supervise the administration of the cytotoxic agent as usual. The only difference from norma practice is that the hospital pharmacy sends an invoice for the drug cost to the patient Although this is not perfect, it is satisfactory for the patient who requires a particular treatment that is otherwise unavailable on the NHS and who is willing and able to pay.

Richard Osborne consultant in medical oncology Dorset Cancer Centre, Poole Hospital, Poole, Dorset BH15 2JB

rjosborne@csi.com

1 Richards C, Dingwall R, Watson A. Should NHS patient be allowed to contribute extra money to their care? $B M$ 2001;323:563-5. (9 September.)
Charges for NHS dental care have led to privatisation

EDITOR-Richards et al asked whether patients in the NHS should be allowed to contribute extra money to their care. In NHS dental primary care charges to patients have been high for over 10 years. ${ }^{1}$ NHS patients pay up to $£ 360$ towards just one course of treatment, and these charges now account for $80 \%$ of the full cost of most dental treatments performed in the NHS.

So have these patient charges improved NHS dental care? In theory, they should have, through additional funding, but after only a few years the government deducted any payments from patients from government payments to dentists, leaving us as unpaid tax collectors with no more funding and a lot more extra paperwork.

With many patients paying such high NHS charges, it was, however, a small step to ask patients to pay the full costs, or to join a low cost plan at about $£ 12$ per month to receive the high quality private dentistry that the government is unprepared to allow or fund properly in the NHS.

So additional patient charges are a good thing-they help the public to start "valuing" medical services while initially providing some additional funds. If the government stays true to form and starts to count charges to patients as government funds, deducting these from grants, etc, then at least offering private quality care will be one step nearer for the public

Modern patient care must come before thinking like a third world government, even if that means funding care outside the NHS. This is now well established in dental care in the United Kingdom, and medicine would do well to learn from NHS dentistry's problems and solutions.

Tony Kilcoyne dental practitioner

Dental Practice, Haworth, West Yorkshire

BD22 8NI

care@smilespecialist.co.uk

1 Richards C, Dingwall R, Watson A. Should NHS patient be allowed to contribute extra money to their care? $B M$ 2001;323:563-5. (9 September.)

\section{Screening for Down's syndrome}

\section{Antenatal screening has human costs}

EDITOR-In their study Gilbert et al made no provision for the cost of counselling. ${ }^{1}$ I still hear from women who go for antenatal care and are screened without consent or counselling. ${ }^{2}$ Some of these women regret entering a conveyor belt process that ends up with an invasive procedure that causes a high rate of fetal loss. Gilbert et al, by highlighting the economic implications of screening, remind us that mothers and their unborn babies continue to be used in what is primarily a cost saving procedure.

Doubt has already been cast upon the public health benefits of screening and the ethical component of such programmes. ${ }^{3}$ No pregnancy is replaceable. The cost of 
screening in human terms has not been evaluated. By excluding the costs of counselling and disregarding mothers' rights and the worth and value to society of children with Down's syndrome, the study by Gilbert et al has severe limitations that should prevent us from embracing such screening strategies.

Josephine Venn-Treloar general practice assistant The Surgery, Welling, Kent DA16 2JZ atreloar@globalnet.co.uk

1 Gilbert RE, Augood C, Gupta R, Ades AE, Logan S, Sculpher M, et al. Screening for Down's syndrome: effects, afety, and cost effectiveness of first and second trimester strategies. BMJ 2001;323:423-5. (25 August.)

$2 \mathrm{~J}$ Venn-Treloar J. Nuchal translucency-screening withou consent. BMJ 1998;316:1026.

3 C Ford. The value of screening for Down's Syndrome in a socio-economically deprived area with a high ethnic population. Br J Obstet Gynaecol 1998;105:855-9.

\section{Costings are crucial to model}

EDITOR-Gilbert et al in their paper provide an analysis of cost and effectiveness of screening for Down's syndrome using mathematical modelling. ${ }^{1}$ Costing of procedures undertaken in the NHS is difficult, and Gilbert et al conclude that four screening strategies are cost effective and efficient.

Nuchal translucency screening is apparently the cheapest procedure, at $£ 4.40$ per test, but there is no indication how this figure was derived. In a cost analysis undertaken for ultrasound scanning for the Royal College of Obstetrics and Gynaecology in 1995, the cheapest scan cost about $£ 35$. This figure was probably unreliable, but the discrepancy is large, and it is important to resolve it since the costs Gilbert et al quote for nuchal translucency screening are crucial to their model. If the costing is inaccurate then the conclusions of the study are likely to be incorrect.

The literature generally agrees that nuchal translucency screening together with serum testing seems an efficient screening method, which is confirmed by this model. But the practical problems that surround implementation of such a programme nationally are formidable. Only about $7 \%$ of units are offering nuchal translucency screening. There are well recognised shortages of radiography staff and possibly inadequate facilities in some circumstances. Early screening demands effective counselling before a woman gets to the hospital, so she has time to consider her options.

One of the benefits of an early diagnosis is a surgical termination, but in this model only about one third of women had their diagnosis in time. Early diagnosis also increases the demand for chorionic villus sampling, which is generally available only in fetal medicine centres, carries a higher miscarriage rate than amniocentesis and incurs higher laboratory costs. Currently about $60 \%$ of women in the United Kingdom are offered at least a double test.

Gilbert et al indicate the weakness of that strategy, but it is a starting point, and the addition of other analytes would not necessarily be difficult. Screening is likely to be more feasible to support during the second trimester than during the first, mainly because of the difficulties of implementing nuchal translucency measurements. This model suggests that a quadruple test may prove effective, and, although there would be an increased cost, the test entails automated, laboratory techniques and not those dependent on a skill-such as nuchal translucency screening. The challenge is to find a technique that is not only cost effective and safe but that is also feasible to implement nationally.

Martin Whittle chairman, antenatal subgroup of national screening committee

Department of Fetal Medicine, Birmingham Women's Hospital, Birmingham B15 2TG m.j.whittle@bham.ac.uk

1 Gilbert RE, Augood C, Gupta R, Ades AE, Logan S, Sculpher M, et al. Screening for Down's syndrome: effects, pher M, et al. Screening for Down's syndrome: effects, strategies. BMJ 2001:323:423-5. (25 August.)

2 Royal College of Obstetrics and Gynaecology. Ultrasound screening for fetal abnormalities. Report of the RCOG Working screening for fetal abnormalities.

\section{Conclusions do not reflect reality}

EDITOR-Gilbert et al have tried to identify the best approach for screening for Down's syndrome. ${ }^{1}$ They have, however, fallen into the trap of uncritically evaluating the published literature. There is no statistical proof that the detection rate is significantly better for any of triple screening versus double screening, quadruple screening versus double or triple screening, or screening in the first trimester versus the second trimester, or that the integrated test is an improvement

All studies of Down's syndrome screening have had small numbers of examples of pregnancies positive for Down's. Consequently, although false positive rates can be accurately assessed, the detection rate cannot. The confidence intervals about the detection rate are so wide that no one has yet proved that the superiority of one method over another is not entirely due to chance. ${ }^{2}$ It has also been known for a long time that where small numbers have been used to derive a population model that is used to estimate false positive and detection rates, large errors can result from an error in the estimate of the population variance for a single analyte giving rise to a hopelessly optimistic estimate of the effectiveness of a procedure.

Studies of the efficacy of nuchal translucency screening have all been interventional, and the quoted detection rate (of the order of $80 \%$ ) therefore needs to be deflated to account for natural fetal losses. The effect of natural fetal loss is to reduce the detection rate for screening during the first trimester to less than $60 \%$, making it comparable to screening during the second trimester. ${ }^{4}$ In addition, for nuchal translucency measurement, there is a far greater incidence of failure to be able to make a measurement than there is failure of biochemical testing to be able to produce a result, which further depresses the detection rate.

Finally, there are serious statistical doubts about the integrated test and worries about the ethics of withholding early positive results until a second test has been performed. ${ }^{5}$ The unit costs estimated for the different tests are also interesting. How can it be that an ultrasound test requiring 15-20 minutes of hands on technical work is cheaper than a biochemical test, when one person can analyse hundreds of samples per day?

Since the detection rates for nuchal translucency screening are grossly overestimated and the integrated test is expensive and unproved, the conclusion that these two modalities of screening represent the boundary of cost effectiveness cannot be left unchallenged.

Tim Reynolds consultant chemical pathologist Queen's Hospital, Burton-on-Trent, Staffordshire DE13 0RB

tim.reynolds@queens.burtonh-tr.wmids.nhs.uk

1 Gilbert RE, Augood C, Gupta R, Ades AE, Logan S, Sculpher M, et al. Screening for Down's syndrome: effects, safety, and cost effectiveness of first and second trimeste strategies. BMJ 2001;323:423-5. (25 August.)

2 Reynolds TM. Downs syndrome screening: A controversi test with more controversy to come! J Clin Path 2000;53:893-8

3 Reynolds TM. Chapter 4: Screening by test combination: a statistical overview. In: Screening for Down's syndrome. Grudzinskas JG, Chard T, Chapman M, Cuckle H, eds. Cambridge: Cambridge University Press, 1994:47-71.

4 Reynolds T. Antenatal screening for Down's syndrome. Lancet 1998:352;1145.

5 Reynolds T, Zimmermann R, Wright E. Integrated screening for Down's syndrome. New Engl J Med 1999:341; $1395-7$

\section{Modelling does not predict reality accurately}

EDitor-Gilbert et al conclude that, of the antenatal screening tests for Down's syndrome, the integrated test is the most effective and safest strategy and that all other strategies result in more liveborn babies or miscarriages of unaffected pregnancies. ${ }^{1}$ Their conclusions are derived from mathematical modelling rather than trial data. This is particularly true when evaluating the integrated test, which has never been tested in a controlled trial nor even in a large uncontrolled demonstration project.

It is difficult to think of any other area of medicine where health policy is so driven by modelling without the support of properly gathered clinical evidence. There are no controlled studies showing that serum or nuchal translucency screening is more effective than maternal age or reduces the rates at which invasive procedures are performed. By contrast, there is clear evidence that modelling does not accurately predict the effectiveness of screening programmes in practice. We reported a $68 \%$ antenatal detection rate over six years, screening by maternal age where routine anomaly scanning was also offered. ${ }^{2}$ Ford et al found that serum screening detected only $31 \%$ of cases of Down's syndrome antenatally. ${ }^{3}$ The modelling relied on by Gilbert predicts that in the first study the detection rate should have been $32 \%$ and in the second $60 \%$.

Modelling does not predict reality accurately for two reasons.

Firstly, women do not behave as predicted. In Ford's study only $71 \%$ accepted screening, so only 13 of 19 cases occurred in those screened.' Only 11 of these 13 were screen positive, of which only six had invasive testing. Only four of the six chose 
termination. In other studies many older mothers decline screening in favour of a definitive diagnostic test, increasing the amniocentesis rate above that predicted.

Secondly, modelling also fails because of inaccurate assumptions about the age structure of the antenatal population. The models assume that $5 \%$ of pregnant women are aged over 35 whereas nationally the proportion is $16.5 \%$ (launch of National Sentinel Caesarean Section Audit). In Southampton this resulted in over $65 \%$ of cases of Down's arising in older women. ${ }^{2}$

Finally, Gilbert et al do not consider some of the negative impact of screening. Women increasingly complain that midwives and doctors are not interested in their pregnancies until they have ruled out Down's syndrome. Age based screening need be discussed with only a small segment of the population, who are often aware when planning their pregnancy of the higher risk of Down's. If we are to retain scientific credibility the choice of the best screening policy should be made on the basis of evidence from controlled clinical trials and not models that are demonstrably inaccurate.

David Howe consultant in fetomaternal medicine Wessex Fetal Medicine Unit, Princess Anne Hospital, Southampton SO16 5YA

1 Gilbert RE, Augood C, Gupta R, Ades AE, Logan S, Sculpher M, et al Screening for Down's syndrome: effects, safety, and cost effectiveness of first and second trimester strategies. BMI 2001;323:423-5. (25 August)

2 Howe DT, Gornall R, Wellesley D, Boyle T, Barber J. Six year survey of screening for Down's syndrome by maternal year survey of screening for Down's syndrome by maternal
age and mid-trimester ultrasound scans. BMJ 2000;320: age and

3 Ford C, Moore AJ, Jordan PA, Bartlett WA, Wyldes MP, Jones AF, et al. The value of screening for Down's syndrome in a socioeconomically deprived area with a high ethnic population. Br J Obstetr Gynaecol 1998;105: $855-9$

\section{Authors' reply}

EDITOR-The questions about costs raised by Venn-Treloar, Whittle, and Reynolds were addressed in the full text version of the report on bmj.com, with further details in the technical report (www.ich.ucl.ac.uk/srtu/ frampubs.htm). ${ }^{1}$ We included the costs of counselling before amniocentesis, chorionic villus sampling, or termination, but we assumed that screening options were discussed with all women at booking. As all women were assumed to have had a dating ultrasound scan, the cost of the nuchal fold translucency test relates to the additional time to take measurements, explain the results, and train ultrasonographers.

Reynolds seems to have missed the section in the methods that explains that the nuchal fold measurement was adjusted for verification bias. Howe makes the case for a modelling exercise. Differences between our detection rates and those from studies based on routine care will be strongly affected by uptake rates, referral practices, and verification bias. Modelling takes account of these factors to allow comparison of test performance and would still be required even if trials were feasible.

Finally, Reynolds raises an important point about the poor precision of the detection rate. One approach is to look for consistency of the characteristics of test performance. Meta-analyses of the results for biochemical markers produce comparatively precise results, which are consistent with the characteristics used in the analysis. But to take account of the correlation between markers we used test characteristics from a single large, archived dataset. Other archived datasets have given similar results.' We believe that this approach gives the best estimates of test performance but accept that random error is not represented.

R E Gilbert senior lecturer in clinical epidemiology C Augood research fellow in systematic reviews $\mathbf{R}$ Gupta research assistant in statistics S Logan senior lecturer in epidemiology Systematic Reviews Training Unit, Department of Paediatric Epidemiology and Biostatistics,

A E Ades reader in biostatistics

Department of Paediatric Epidemiology and Biostatistics, Institute of Child Health, London WC1N 1EH

M Sculpher senior research fellow

Centre for Health Economics, University of York, Heslington, York YO1 5DD

J H P van der Meulen senior lecturer in clinical epidemiology

Health Services Research Unit, Department of Public Health and Policy, London School of Hygiene and Tropical Medicine, London WCIE 7HT

1 Gilbert RE, Augood C, Gupta R, Ades AE, Logan S, Sculpher M, et al Screening for Down's syndrome: effects, strategies. BMJ 2001;323:423-5. (25 August.)

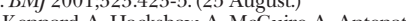
. Kennard A, Haw screening for Down's syndrome. J Med Screen 1997;4:181-

Ratio of femoral length to tibial length needs to be evaluated extensively

EDITOR-Gilbert et al and Howe et al in their articles rely heavily on maternal age to screen for Down's syndrome. ${ }^{12}$ But materna age is not so useful in India and other countries where early marriage is the norm and the social pressures for early motherhood are enormous. In our series, which included 3000 deliveries and seven babies with Down's syndrome, we saw that all babies with the syndrome were born to mothers younger 35 years.

We reported in the American Journal of Perinatology our finding that the ratio of femoral length to tibial length remains remarkably constant around 1.15 (range 1.13-1.19) in fetuses after 13 weeks' gestation. Fetuses with Down's syndrome had this ratio greater than 1.2 (standard deviation 4.5) compared with norms. The youngest fetus with Down's syndrome in our sample was 22 weeks old at the time of measuring. We hope that this ratio will be evaluated more extensively and earlier in pregnancy, to see if these findings are valid in the early second trimester and across ethnic groups.

Pooja Sachdev registrar in paediatric Shubhra Bahl senior house officer in paediatric Jacob M Puliyel consultant paediatrician puliyel@vsnl.com

Department of Pediatrics, St Stephen's Hospital,

Tis Hazari, Delhi 110054, India

1 Gilbert RE, Augood C, Gupta R, Ades AE, Logan S, Sculpher M, et al. Screening for Down's syndrome: effects, safety, and cost effectiveness of first and second trimester safety, and cost effectiveness of first and second
strategies. BMJ 2001;323:423-5. (25 August.)
2 Howe DT, Gornall R, Wellesley D, Boyle T, Barber J. Six years survey of screening for Down's syndrome by maternal age and mid trimester ultrasound scans. BMJ 2000;320 606-10.

3 Gupta R, Thomas RD, Sreenivas V, Walter S, Puliyel JM. Ultrasonographic femur-tibial length ratio: a marker of Down syndrome from the late second trimester. Am J Perinatology 2001;18:217-23

Nuchal translucency screening may help detect congenital heart disease

EDITOR-Screening for Down's syndrome during fetal life has received much attention, with over 350 articles in the medical literature over the past five years alone. The data on cost effectiveness provided by Gilbert et al are welcome, given the variable screening strategies offered by different NHS providers. ${ }^{1}$ Increased nuchal translucency may be a marker of many fetal diseases, and a discussion of its cost effectiveness should not be restricted to Down's syndrome alone.

Congenital heart disease is the most common type of fetal malformation (at least $8 / 1000$ during fetal life), but only $25 \%$ of clinically significant malformations are actually detected prenatally in the United Kingdom, ${ }^{2}$ which is worse than any other type of fetal anomaly. Increased nuchal translucency is associated with congenital heart disease, independent of karyotypic abnormalities, with a sensitivity of $15-56 \% .^{3-5}$ Although far from perfect as a screening tool for congenital heart disease, nuchal scanning makes some contribution to prenatal detection of congenital heart disease and chromosomal abnormalities. This is highly relevant when the relative merits and cost effectiveness of prenatal screening strategies are evaluated.

John M Simpson consultant in fetal and paediatric cardiology

Department of Congenital Heart Disease,

Guy's Hospital, London SE1 9RT

john.simpson@gstt.sthames.nhs.uk

1 Gilbert RE, Augood C, Gupta R, Ades AE, Logan S, Sculpher M, et al. Screening for Down's syndrome: effects, safety, and cost effectiveness of first and second trimeste strategies. BMJ 2001;323:423-5. (25 August.)

2 Bull C. Current and potential impact of fetal diagnosis on prevalence and spectrum of serious congenital heart disease at term in the UK. British Paediatric Cardiac Association. Lancet 1999;354:1242-7.

3 Mavrides E, Cobian-Sanchez F, Tekay A, Moscoso G, Campbell S, Thilaganathan B, et al. Limitations of using first-trimester nuchal translucency measurement in routine screening for major congenital heart defects Ultrasound Obstet Gynecol 2001;17:106-10.

4 Michailidis GD, Economides DI Nuchal translucency Michailidis GD, Economides DL. Nuchal translucency measurement and pregnancy outcome in karyotypical

5 Hyet J Per.

Hett J, Perdu M, Sharland G, Snjders R, Nicolaides KH. Using fetal nuchal translucency to screen for major congenital defects at 10-14 weeks of gestation: populatio
based cohort study. BMJ 1999;318:81-5.

\section{Problems with temporary cardiac pacing}

\section{Ultrasonography can aid central venous cannulation}

EDITOR-Murphy in his editorial highlights problems with temporary cardiac pacing where the service is provided by doctors in training with inadequate supervision. ${ }^{1}$ An essential component of temporary pacing is obtaining central venous access. The failure 
rate for this intervention remains notable. Murphy suggests involving anaesthetists or intensivists to help resolve this problem.

There are other ways to address this important issue rather than increasing the workload of another department. The first would be to increase the involvement of consultant physicians from the current level of only $14 \%$. Failure rates for central venous cannulation are related to the experience of the operator, with failure rates almost double in inexperienced hands. ${ }^{2}$

The second is to move from a landmark guided technique for central venous cannulation to an ultrasound guided technique. A meta-analysis comparing ultrasound guidance with a landmark technique for central venous cannulation suggested that ultrasound guidance significantly reduced the rates of failures and complications and the need for multiple attempts at placement. ${ }^{3}$ The benefits may be more obvious in patients with difficult central venous access. ${ }^{4}$

Paul Jefferson consultant in anaesthesia and intensive care medicine

p.jefferson@dgri.scot.nhs.uk

Vincent Perkins consultant in anaesthesia and intensive care medicine

Dumfries and Galloway Royal Infirmary, Dumfries DG1 4AP

1 Murphy JJ. Problems with temporary cardiac pacing. $B M$ J 2001;323:527. (9 September.)

2 Sznajder JI, Zveibil FR, Bitterman H, Weiner P, Bursztein S. Central vein catheterization. Failure and complication rates by three percutaneous approaches. Arch Intern Med 1986;146:259-61.

3 Randolph AG, Cook DJ, Gonzales CA, Pribble CG. Ultrasound guidance for placement of central venous catheters: A meta-analysis of the literature. Crit Care Med 1996;24:2053-8

4 Hatfield A, Bodenham A. Portable ultrasound for difficul central venous access. BJA 1999;82:822-6.

\section{Better training in general medicine is} required

EDITOR-Temporary transvenous cardiac pacing is a life saving procedure and an important skill for emergency physicians to have. ${ }^{1}$ It is often a poorly taught procedure with potentially life threatening complications. It should not be embarked on without due care, attention, and training. When possible, waiting and observing more stable patients overnight until the permanent pacemaker list is much more desirable. But potentially deskilling the frontline may have equally disastrous consequences, as would transferring potentially unstable patients to other centres.

If trainees in general medicine are not to expected to perform temporary pacing, then should the same be said for chest drains, central lines, and lumbar punctures? When specialty medicine does cover 24 hours patients will expect specialists performing their particular skill, but further deskilling general medical trainees in the current climate may do more harm than good. What we need is better training in general medicine or an overhaul of acute medicine.

Tristan Richardson specialist registrar Royal Bournemouth Hospital, Bourenmouth BH7 7DW

tilrichardson@hotmail.com

Murphy JJ Problems with temporary cardiac pacing. BMJ 2001;323:527. (9 September.)

\section{Training is essential}

EDITOR-I was surprised to read in the editorial by Murphy that one of his proposed solutions to the problems with temporary cardiac pacing was to ask anaesthetists to step in to address a deficiency in general medical training. ${ }^{1}$ Central venous cannulation is an oft performed and sometimes life saving procedure, and lack of competence in this technique among physicians in training suggests that training needs to be changed.

With recent changes in training in anaesthetic and intensive care medicine there is an increasing number of preregistration training posts in anaesthesia. A growing number of senior house officer posts in intensive care medicine is also available to trainees from all medical backgrounds where competency based training in a wide range of procedures, including placement of central lines, is offered. Failing this almost all acute hospitals have some form of critical care unit where trainees and senior physicians are likely to be welcomed in order to attain and maintain competency in such procedures. Taking advantage of such opportunities is surely a better way to address training issues while encouraging the development of even closer cooperation between the two specialties.

Sophie Risebero consultant anaesthetist Department of Anaesthetics, Worcester Royal Infirmary, Worcester WR5 1HN srisebero@hotmail.com

\section{Murphy JJ. Problems with temporary cardiac pacing. $B M$ 2001;323:527. (9 September.)}

Formal training in core procedure is lacking for specialist registrars in general internal medicine

EDitoR-Murphy draws attention to the deficiencies in the training of general physicians (non-cardiology) in temporary cardiac pacing. ${ }^{1}$ The Joint Committee for Higher Medical Training of the Royal College of Physicians has stated that training in procedure is mandatory for accreditation in general (internal) medicine by including it as one of the "core/essential" procedures of the curriculum for specialist registrars in this specialty. In the absence of formal training, acquisition and maintenance of competence at temporary cardiac pacing requires opportunity to perform the procedure. We therefore used a questionnaire to determine specialist registrars' experience in this technique.

Twenty specialist registrars seeking dual accreditation in a chosen specialty and general internal medicine who participated regularly in the general medical on call rotas (18 specialist registrars) or the intensive care (two) of a 1000 bed teaching hospital answered a series of questions about their experience in temporary cardiac pacing.

The number of temporary pacemaker wire insertions performed by the registrars varied greatly. Four had inserted between 21 and 50 temporary wires, the chosen specialty of two of them being intensive care medicine. Fifteen had inserted fewer than 10 temporary pacing wires (supervised and unsupervised) during their professional career, seven had inserted fewer than five, and one had not inserted any.

Although most of these specialist registrars had some experience of temporary cardiac pacing, few were currently performing the procedure often enough to maintain competence. Only three of the group had inserted a temporary pacing wire within the previous 6 months. Seven had performed the procedure within the previous 12 months, six between 1 and 2 years previously, and three more than 2 years previously, with one specialist registrar not having inserted a temporary wire in the previous 5 years. Ten of the group thought that they would like to have a more experienced member of staff to help them in case of difficulty.

Specialist registrars seeking accreditation in general internal medicine are currently the most senior doctors in house to provide temporary cardiac pacing. Furthermore, they are expected to provide guidance and training in this procedure to more junior medical colleagues and subsequently assume overall responsibility as general medical consultants on call. Our study shows that most specialist registrars do not perform temporary cardiac pacing often enough to maintain or even achieve competence in this procedure. Formal training in this procedure therefore will be necessary if it is to remain as a core/essential procedure required of practitioners of general internal medicine.

Liam Cormican specialist registrar in respiratory medicine and general internal medicine

Department of Respiratory Medicine, Thomas Guy House, Guy's Hospital, London SE1 9RT cormitron@yahoo.com

Emma Baker senior lecturer (honorary consultant) Department of Physiological Medicine, St George's Hospital Medical School, London SW17 0RE

1 Murphy JJ. Problems with temporary cardiac pacing. BMJ 2001;323:527. (9 September.)

Novices can reliably and safely perform temporary pacing from femoral route

EDITOR-A need for urgent temporary pacing remains in district general hospitals. External cardiac pacing is useful but is not always reliable and can be quite painful. Transferring patients to a specialist centre with recurrent syncope or significant hypotension, regardless of the use of isoprenaline infusions and external pacing is not acceptable

What is required is a straightforward and reliable technique of transvenous temporary pacing that can be performed by a senior house officer, specialist registrar, or consultant with no or little recent experience. The two possibilities are balloon flotation catheters inserted through the subclavian or jugular vein and semirigid pacing wires through the femoral vein. Balloon flotation catheters work well and can be inserted by a novice with the aid of a simple instruction diagram, assuming that central access is achieved. ${ }^{2}$ Traversing the tricuspid valve can, however, be difficult in the presence of severe tricuspid regurgitation. 
The ideal solution for urgent transvenous temporary pacing by a novice is the femoral route and a standard wire. The anatomical landmarks are well defined, and access is reliably achieved. External compression on the femoral vein allows haemostasis if bleeding complicates thrombolysis. The temporary wire readily crosses the tricuspid valve to take up a stable position, even in the presence of severe tricuspid reflux. Little manipulation is required within the heart because the wire follows a natural curve. This minimises procedure time and the incidence of ventricular arrhythmias. If there are concerns regarding incidence of infection the wire can be renewed with a new wire from the subclavian or jugular as a planned exercise allowing the possibility for teaching.

We conducted a feasibility study over a period of eight months to assess the ease with which senior house officers who had no or very little pacing experience could undertake femoral route pacing when instructed by a specialist registrar in cardiology (group A, 17 patients). A different senior house officer was invited to perform the procedure each time after a brief explanation. All achieved access without complication and a suitable pacing wire position under the guidance of the specialist registrar. This group was compared with the remainder undergoing temporary pacing, performed by more experienced senior house officers alone or with supervision by their non-cardiology consultant (group B, 13 patients). Most in group B used jugular or subclavian access. Ethics approval was not sought because the study was a retrospective comparison of two groups undergoing a clinically indicated procedure by equally accepted techniques.

In group A, 1 of the 17 patients $(6 \%)$ required repositioning and $10(59 \%)$ were placed under six minutes compared with 3 $(23 \%)$ and $6(46 \%)$ of the 13 patients in group B, respectively. Temporary pacing via the femoral vein is reliable, safe, and quickly learnt by novices.

Nigel Clarke cardiology specialist registrar nigelra.clarke@btinternet.com

Patrick Davey consultant cardiologist David Sprigings consultant cardiologist

John Birkhead consultant cardiologist

Northampton General Hospital, Northampton

NN1 5BD

1 Murphy JJ. Problems with temporary cardiac pacing. BMJ 2001:323:527. (9 September.)

2 Fergusson JD, Banning AP, Bashir Y Randomised trial of temporary cardiac pacing with semirigid and balloonfloatation catheters. Lancet 1997;349:1883.

\section{Mental health services for children and adolescents}

\section{Raised profile of child health services is welcome}

EDITOR-The announcement of Professor Aynsley-Green's appointment as national director for children's healthcare services, follows on the important government decision to establish a national service framework for children's services, announced last year. ${ }^{1}$ It is around 18 months since the $B M J$ published an article by Aynsley-Green et al pointing to the neglect of children at a policy level.

This neglect applies in a great measure to the mental health services for children and adolescents. These services-despite recent much heralded ringfenced modernisation monies, now discontinued after only two years-have long waiting lists for outpatient services and inadequate numbers of beds for mentally ill young people, who end up inappropriately on adult wards and paediatric units.

Child mental health professionals have been encouraged by the decision of the Health Secretary, Alan Milburn, to include child and adolescent mental health services, along with maternity and social care services, within the national service framework. These services were excluded from the recent national service framework for mental health. Most regard the framework for children's services as the proper place to consider the changes to strategy, workforce, structures, and resources that are urgently needed to meet the mental health needs of the nation's children. A study conducted by the Office for National Statistics showed that $10 \%$ of children and young people in England and Wales have an identifiable mental health disorder. The commonest conduct disorder, reported by Scott et al to be amenable to parenting training, costs the patient, his family, and society dear-in economic terms, by the age of 28 years, 10.00 times higher than those with no problems. ${ }^{3}$ Not all these young people with mental health disorder need or will access specialist mental health services for children and adolescents. But the findings underline the importance of creating health, education, and social care services that, together with the communities in which our families live, will be appropriately funded to combat stigma, promote emotional wellbeing, and address the hitherto unmet mental health needs of the children.

Caroline Lindsey consultant child and adolescent psychiatrist

Child and Family Department, Tavistock Clinic, London NW3 5BA

clindsey@tavi-port.org

1 White C. New child tsar promises shake-up of services. BMJ 2001;323:302. (11 August)

Aynsley-Green A, Barker M, Burr S, Macfarlane A, Morgan $\mathrm{J}$, Sibert J, et al. Who is speaking for children and adolescents and for their health at the policy level? BMJ adolescents and for

3 Scott S, Spender Q, Doolan M, Jacobs B, Aspland H. Multicentre controlled trial of parenting groups for childhood centre controlled trial of parenting groups for childhood
antisocial behaviour in clinical practice. BMJ 2001;323. 194. (28 July)

\section{Titles are ridiculous}

EDITOR-Our masters, it seems, have appointed yet another medical tsar. ${ }^{1}$ It is odd that they have yet to appoint a tsarina; even odder that they have "gone foreign" to find an unsuitable title. The tsars, after all, were absolutist Russian rulers whose behaviour, on occasion, was not what we would expect in our caring health service. I thought that a nation so rich in ridiculous titles could have devised something more appropriate and British, such as "paediatrician-in-waiting to the minister's bedchamber."

If the men in suits were determined to add a touch of Eastern exotica to our governance, they could have turned to their childhood story books and given us a grand vizier or two. But once you start delving into story books, you realise the most appropriate title might be grand panjandrum.

Michael O'Donnell writer and broadcaster Handon Cottage, Loxhill, Godalming, Surrey GU8 4BD

michael@odonnell99.freeserve.co.uk

1 White C. New child tsar promises shake-up of services. BMJ 2001;323:302. (11 August.)

\section{Fabricated or induced illness in children}

\section{Open mind is needed regarding origins of childhood symptoms and illnesses}

EDITOR-I do not agree with Wilson that guidance issued by the Department of Health for consultation is excellent. ${ }^{1}$ Wilson admits that the origins of Munchausen syndrome by proxy were based in the personal experience of Roy Meadows.

Is this how paediatric diagnoses are made, simply on the basis of an individual's subjective experience? Especially when such a "diagnosis" has had such a devastating and damaging effect on many hundreds of children and families in the United Kingdom and around the world. Emotional damage is caused to children, their parents, and siblings from being separated and placed into state care; or where stigmatised parents, avoid contact with doctors because they would be dismissed as child abusers if they presented their children for medical treatment. Consequently some very ill children have not received the medical attention they so desperately needed.

So, Munchausen by proxy is not based on painstaking research under scientific conditions? There is no system of verification nor validation by an accredited national medical body? No field testing has been done over a reasonable period of time? Does the Royal College of Paediatrics and Child Health not have a duty and responsibility to all children and parents to ensure that paediatric diagnoses are not merely based on the subjective personal experience of one paediatrician?.

Wilson also says that a child's doctor is not required to clarify whether inappropriate parental care is the result of mental illness, and yet there are doctors without a psychiatric qualification who have stated in evidence to courts in cases of Munchausen by proxy that the parent has a serious personality disorder.

The claim by Wilson that rates of fabricated or induced illness in children are underestimates is not based in fact. If it were, then there must be many thousands of extremely gullible doctors in the United 
Kingdom who can so easily be deceived, and their ability to diagnose childhood illnesses is seriously impaired.

I agree with Wilson that we need to have an open mind regarding the complex origins of childhood symptoms and illnesses, but unfortunately the Department of Health's guidance and Wilson's letter do not show such open mindedness.

Charles Pragnell social care management consultant Social Care Consultants, Redcar and Cleveland TS10 2JN

1 Wilson R. Fabricated or induced illness in children. $B M$ J 2001;323;296-7. (11 August.)

\section{Author's reply}

EDITOR-Pragnell and his work are motivated by his sense of injustice done to some parents. His remarks have no clear target, and he seems to wish to support individuals by claiming that fabricated or induced illness does not exist. He wishes to protect parents; I wish to protect children first. $\mathrm{He}$ seems to have a punitive retrospective aim. Professionals and the Department of Health, as well as the overwhelming majority of the public, want preventive and protective action to improve the present and the future. The Royal College of Paediatrics and Child Health and Department of Health have used the views of children and families in drawing up their current procedures.

I know that Pragnell wants progress too. Rather than be attracted by the flames of conflict we should highlight the issues on which we agree. This may be easier when the full draft of the royal college's document is issued.

Richard Wilson consultant paediatrician Kingston NHS Trust, Kingston KT2 7AZ

\section{Endometriosis}

\section{Emphasis on medical treatment is misleading}

EDITOR-In his review on endometriosis, Prentice sets out management strategies for treating painful symptoms resulting from endometriosis. ${ }^{1}$ He concludes that the first line treatment should be medical and that surgery should be reserved for cases in which medical treatment has failed or for patients with severe disease. We believe that the emphasis on medical treatment and the assertion that controversy exists over the precise role of surgery are misleading and may be responsible for physicians mismanaging their patients.

The evidence from randomised controlled trials for the medical and surgical management of endometriosis has recently been reviewed. ${ }^{2}$ A critical summary of the medical management has shown that there is little difference in effectiveness of various medical treatments, which only last while patients remain on treatment. The review also showed that surgical management is effective in the treatment of both painful symptoms and subfertility. Furthermore, there is a wealth of grade II and III evidence in the literature which supports laparoscopic surgery as the primary treatment modality for all stages of endometriosis. ${ }^{3}$

We believe that in the United Kingdom endometriosis is misdiagnosed in many patients who are having a laparoscopy, and many are not treated adequately because of the emphasis on medical management strategies. ${ }^{3}$ This almost certainly reflects the fact that only a few surgeons in the United Kingdom have the advanced endoscopy skills required to diagnose and then treat the disease laparoscopically. This is in marked contrast to the situation in the rest of Europe and North America, where surgical techniques using minimal access were first developed.

We recommend that medical treatments be used by primary care doctors for the short to medium term to control painful symptoms, before referral to hospital for surgery or while the patient is on the waiting list for surgery. Patients should be referred to units where laparoscopic diagnosis and surgery can be carried out during the same operation. Conservative, excisional, or ablative endoscopic surgery and not medical treatment should be the first line management of patients with endometriosis. This applies to women with any stage of the disease, but particularly those with endometriotic cysts, or infiltrating rectovaginal disease. ${ }^{45}$

Kevin D Jones clinical fellow in gynaecological endoscopic surgery

Christopher Sutton consultant gynaecologist Royal Surrey County Hospital, Guildford GU2 5XX

1 Prentice A. Endometriosis. Regular review. BMJ 2001;323: 93-5. (14 July.)

2 Farquhar C, Sutton CJG. The evidence for the management of endometriosis. Curr Opin Obstet Gynaecol 1998;10 ment of

3 Garry R. Endometriosis: an invasive disease. Gynaecol Endoscopy 2001;10:79-83.

4 Jones KD, Sutton CJG. Laparoscopic management of ovarian endometriomas: a critical review of curren practice. Curr Opin Obstet Gynaecol 2000;12:309-17.

5 Jones KD, Sutton CJG. Arcus taurinus: the mother an father of all LUNAs. Gynaecol Endoscopy 2001;10:83-91.

Clinicians and patients should be aware of association between endometriosis and malignancies

EDIToR-Prentice's review highlights the difficulties encountered in clinical practice in relation to endometriosis. ${ }^{1}$ Clinicians should use abdominal or transvaginal ultrasound imaging of the pelvis when women present with pelvic pain, dysmenorrhoea, or dyspareunia to ascertain whether the ovaries are normal or contain endometriotic cysts. The presence of these cysts may indicate more widespread disease in the pelvis. This is important for women about to undergo major gynaecological surgery as unexpected extensive and severe endometriosis may lead to more surgery than had been discussed preoperatively or consented to by the patient. This has medicolegal implications.

Another issue is that of the association between endometriosis and ovarian cancer
So far this has not been a subject that is discussed in full with patients. But a study of 1000 cases of endometriosis reported that, in $8.8 \%$ of cases, malignancy and endometriosis were both present in the same organ, but not necessarily microscopically contiguous, and higher if the lesions were located in different organs. ${ }^{2}$ Malignancies were also more commonly found in ovaries that contained endometriosis. Clear cell and endometroid carcinomas were the malignancies most commonly seen in endometriotic ovaries, and there was clear association between these histological types and endometriosis. Clinicians and patients must be made aware of this association, especially when surgery is contemplated in comparatively young women.

Nazar N Amso senior lecturer and honorary consultant obstetrics and gynaecology University Hospital of Wales, Cardiff, CF14 4XN amsonn@cf.ac.uk

Competing interests: None declared.

1 Prentice A. Endometriosis. Regular review. BMJ 2001;323: 93-5. (14 July.)

2 Stern RC, Dash R, Bentley RC, Snyder MJ, Haney AF, Robboy SJ. Malignancy in endometriosis: frequency and comparison of ovarian and extriarian types. Pathol 2001;20:133-9.

\section{Media could be used to better effect than inducing fear}

EDITOR-Lightfoot et al discussed responses to bioterrorist threats. ${ }^{1}$ One of the aspects that I found most interesting about the recent anthrax releases in the United States is the apparently disproportionate fear that they have produced among the population, fuelled, I think, by the media coverage they have been given.

According to a report published by the US Centers for Disease Control and Prevention on 25 September 2001, in 1999, 28874 people died of firearms injuries, 19102 of drug related causes, and 19171 of alcohol related causes. Neither the statistics on drugs deaths nor those on alcohol related deaths include accidents, homicides, or other causes indirectly related to their use. Given that the media are able to wield such massive influence, perhaps in the hoped for "new world order" they can do so in more useful ways.

Tim Kerruish consultant, emergency medicine Emergency Department, Dunedin Hospital, 201 Great King Street, Private Bag 1921, Dunedin, New Zealand

kerruish@voyager.co.nz

1 Lightfoot N, Wale M, Spencer R, Nicoll A. Appropriate responses to bioterrorist threats. BMJ 2001;323:877-8. (20 October.)

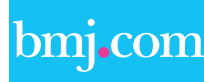

\section{Rapid responses}

Correspondence submitted electronically is available on our website 